\title{
Proteomics as a Tool for Understanding Schizophrenia
}

\author{
Daniel Martins-de-Souza \\ Max Planck Institute of Psychiatry, Munich, Germany and Lab. de Neurociências (LIM-27), Inst. Psiquiatria, Fac. de Medicina da \\ Universidade de São Paulo, São Paulo, Brazil
}

\begin{abstract}
Schizophrenia is likely to be a multifactorial disorder, consequence of alterations in gene and protein expression since the neurodevelopment that together to environmental factors will trigger the establishment of the disease. In the post-genomic era, proteomics has emerged as a promising strategy for revealing disease and treatment biomarkers as well as a tool for the comprehension of the mechanisms of schizophrenia pathobiology. Here, there is a discussion of the potential pathways and structures that are compromised in schizophrenia according to proteomic findings while studying five distinct brain regions of post-mortem tissue from schizophrenia patients and controls. Proteins involved in energy metabolism, calcium homeostasis, myelinization, and cytoskeleton have been recurrently found to be differentially expressed in schizophrenia brains. These findings may encourage new studies on the understanding of schizophrenia biochemical pathways and even new potential drug targets.
\end{abstract}

KEY WORDS: Schizophrenia; Proteomics; Energy metabolism; Oligodendrocytes; Myelin; Calcium.

\section{EXPLORING SCHIZOPHRENIA BRAIN TISSUE}

Schizophrenia (SCZ) is a chronic, debilitating, psychotic mental disorder that affects about $1 \%$ of the world population and is characterized by a range of positive and negative symptoms. ${ }^{1)}$ It is considered a multifactorial disease, likely to be caused by alterations in gene and protein expression since the neurodevelopment combined with environmental factors. The genetic predisposition seems to be pivotal to the establishment of the disorder. ${ }^{2)}$

Considering that SCZ is a brain disorder, the study of brain tissue seems a natural path to follow for the understanding of its pathobiology. Some brain regions have been more strongly correlated to $\mathrm{SCZ}$, such as the prefrontal cortex (PFC). ${ }^{3)}$ PFC executes high functions such as working memory, differentiation of conflicting thoughts, differentiation of right and wrong, correct social behavior and personality expression, ${ }^{4)}$ receiving peripheral sensory inputs from the thalamus. The thalamus acts as a relay center and is involved with auditory, somatic, visceral, gustatory, and visual systems as well as regulation of

\footnotetext{
Received: May 27, 2011 / Revised: July 1, 2011

Accepted: July 6, 2011

Address for correspondence: Daniel Martins-de-Souza, PhD Max Planck Institute for Psychiatry, Kraepelinstr. 2, 80804 Munich, Germany

Tel: +49-89-30622-211, Fax: +49-89-30622-610

E-mail: martins@mpipsykl.mpg.de
}

sleep states, wakefulness, and consciousness. ${ }^{5)}$ Significant alterations in cerebral metabolic activity and related circuitry were found in the thalamus of SCZ patients by imaging studies. ${ }^{6}$ )

The anterior cingulate cortex (ACC) is involved in cognitive and autonomic functions. ${ }^{7)}$ Alterations in synaptic connectivity ${ }^{8)}$ and cognitive control ${ }^{9)}$ were previously described in SCZ ACC, supported by cellular and neurochemical changes. ${ }^{10)}$

Functions such as high-level auditory and visual processing, language, and transference from short- to longterm memory which are compromised in SCZ are concentrated in the temporal lobe. Most probably, it involves an imbalance of glutamate and gamma-aminobutyric acid (GABA) leading to dopaminergic dysfunctions. ${ }^{11)}$ In addition, a reduction of the temporal lobe size in SCZ has been shown by several studies with magnetic resonance imaging. ${ }^{12)}$ As part of the temporal lobe, the superior temporal gyrus (STG) is the major brain area related to speech, language and communication, which also seems to be impaired in SCZ. ${ }^{13)}$ The left STG, which present significant differences from the right STG in SCZ, ${ }^{14,15)}$ is formed by the primary auditory cortex and the Wernicke's area (WA), which is an important region for speech processing and language skills.

Thanks to a collaboration with the Brain NetEurope (http://www.brainnet-europe.org) and to the kind comprehension of the donors and their families, the above de- 
scribed brain regions were available to our group. Initially, our main objective was to search for SCZ biomarkers candidates using comparative proteome analyses. We aimed to provide information on the risk for the disease, to contribute to the early diagnosis and to the prediction of therapeutic response. However, while conducting our proteomic studies in several brain regions, it became clear that the secondary objectives of this type of research, which were to provide detailed information about the pathophysiology of the disease and to further confirm the importance of certain biochemical pathways, have produced more useful findings. Although potential protein bimarkers candidates have also been pointed out as primarily expected, confounding factors that the brain tissue samples bring along and the specificity of those markers were challenges we faced. But certainly, proteomics reveal itself as an interesting tool for a better comprehension of the pathobiology of SCZ.

\section{A TOOL CALLED PROTEOMICS}

SCZ biomarkers have been initially searched by genotyping techniques, genome wide association studies and large-scale transcriptome analyses. Afterwards, proteomic analysis has emerged in this context as a promising strategy.

The term "proteome" was defined in the mid-1990's as the study of the total set of expressed proteins by a cell, tissue or organism at a given time under a determined condition. ${ }^{16)}$ This term has given birth to a whole science nowadays known as "proteomics" that includes many other aspects of global protein analyses such as the modifications made to a particular set of proteins produced by an organism or cellular system and how this can vary with time and under the influence of environmental factors. Comparative proteome analysis, which consists in determining differentially expressed proteins usually comparing two or more different states are the most widespread tool used in clinical studies and has been largely employed in SCZ studies. Knowing the set of proteins that are differentially expressed may lead to an integrated view of modifications in protein expression that can lead to the identification of impaired biochemical pathways, providing information about the pathobiology of the disease. Moreover, the identification of impaired pathways may drive drug discovery studies. Depending on the studied tissue and the background of the analyzed samples regarding their confounding factors, the set of differentially expressed proteins reveal by proteomics can be used as a di- agnostic tool or even to monitor the effectiveness of treatment response.

\section{Two-dimensional Gel Electrophoresis}

The most traditional methodology for the study of the proteome is the two-dimensional gel electrophoresis (2DE) created in the 1970's. ${ }^{17)}$ The main concept is to separate proteins in two dimensions - by their charge (isoelectric point) and apparent mass - for having a distinct resolution of the proteome. In SCZ studies, this tool has been extensively used ${ }^{18)}$ for protein separation combined to mass spectrometry (MS) for protein identification. By combining both technologies it is possible to separate and identify simultaneously many hundreds of proteins in a single experiment. The direct comparison of 2DE maps from different samples using computational tools can provide a picture of which proteins are differentially expressed.

Although largely used, 2DE-MS present drawbacks such as a difficulty in detecting proteins with extremes of high or low molecular weight as well as low-abundance and acidic or basic proteins. ${ }^{19)}$

\section{Shotgun Proteomics}

Considering the increasing power of MS methods and equipments, researchers developed proteomic approaches that could skip the use of gel-based proteomics, avoiding then its limitations. ${ }^{20)}$ Such approaches known as shotgun proteomics are generally a combination of chromatographic steps prior to MS analyses in a high-throughput way. ${ }^{21-23)}$ The quantification of the proteome using shotgun proteomics, either using stable-isotope or label-free methods, are more accurate than the 2DE-MS approach. One potential drawback of shotgun approaches is that they are not capable of providing direct information on intact proteins, contrary to $2 \mathrm{DE}$. Shotgun proteomics has also been employed in SCZ studies. ${ }^{18)}$

\section{Metabolomics}

Data generated by comparative proteome analyses of clinical samples - in this case SCZ - may lead to the identification of biochemical pathways and consequently providing indication of the associated metabolites. The quantification of metabolites using diverse approaches such as enzymatic assays, ${ }^{24)}$ high-resolution proton nuclear magnetic resonance spectroscopy (1H-NMR), ${ }^{25)}$ high-performance liquid chromatography (HPLC) ${ }^{26)}$ can provide a more complete picture of brain activities which may be useful for comprehension of the biochemical processes as 
well as a means of providing a source of non-proteinbased biomarkers.

\section{MAIN FINDINGS IN THE BRAIN PROTEOME OF SCHIZOPHRENIA}

Using proteomic analyses, we investigated 5 different brain regions - PFC, WA, ACC, anterior temporal lobe and thalamus ${ }^{24,27-31)}$ - revealing differentially expressed proteins that led us to discover and confirm the impairment of the following pathways and structures.

\section{Energy Metabolism}

The first indication of impairments in the energy metabolism in SCZ patients was described more than 90 years ago. ${ }^{32)}$ The modern technologies allow a closer look to what has been hypothesized so long ago. Kooy observed hyperglycemia in SCZ patients, hypothesizing that the levels of blood sugar may influence the behavior. Proteomics data support this affirmation ${ }^{33)}$ as well as imaging analyses, ${ }^{34)}$ and biochemical techniques ${ }^{35)}$ and hormonal measurements. ${ }^{36}$

Impairments on glucose handling lead to disturbances in the mitochondrial metabolism. Mitochondria contain a very complex network of self-regulating enzyme for optimizing metabolic processes. Surely, impairments in the activity of one or more of these components will result in altered energy production, also leading to the production reactive oxygen species (ROS). Studying proteomics, we could point out the exact enzymes involved on these processes, leading us to a better comprehension of the biochemistry of SCZ and also to the identification of potential drug targets. ${ }^{33)}$

Several enzymes of the glycolysis such as such as hexokinase, triose phosphate isomerase, glyceraldehyde phosphate dehydrogenase, phosphoglyceromutase, enolase, and pyruvate kinase - were found to be differentially expressed in the proteome analyses of SCZ brain tissue. Using a metabolic map as designed by Kyoto Encyclopedia of Genes and Genomes (KEGG) analysis ${ }^{37)}$ (http:// www.genome.jp/kegg) (Fig. 1), we can have a clear look of the influence of glycolysis in SCZ. Compromising glucose metabolism will lead to pivotal alterations in the cellular energy generation. In a tentative of functionally validate the proteomics findings, we quantify relatively the amounts of pyruvate in SCZ compared to controls. In the thalamus of SCZ patients, levels of pyruvate were significantly decreased. ${ }^{24)}$ These findings may lead to therapeutic and pharmacologic targets. Lower levels of pyr- uvate in SCZ brains may compromise Krebs cycle function, as also supported by our proteomics studies considering the differential expression of aconitase 2 and malate dehydrogenase 1. Moreover, higher levels of nicotinamide adenine dinucleotide phosphate (NADPH) have also been found in the thalamus of SCZ patients, ${ }^{24)}$ which can compromise oxidative phosphorylation (OXPHOS). This is also supported by proteomics since components of the mitochondrial complex $\mathrm{I}$ and $\mathrm{V}$ are altered in SCZ brains. ${ }^{18)}$ The overall dysregulation of energy metabolism leads to the increased production of hazardous ROS which can trigger oxidative stress events. This processed was also pointed out by proteomics through the differential expression of peroxiredoxins. Moreover, other proteins indicating oxidative stress such as members of gluthatione transferases family, carbonyl reductase (CBR) 1, CBR3 and quinoid dihydropteridine reductase (QDPR) we found to be differentially expressed. The differential expression of transketolase indicates a dysfunction in the pentose phosphate pathway which main function NADPH for OXPHOS. The different levels of NADPH in thalamus interestingly support this finding. ${ }^{24)}$

\section{Calcium Homeostasis}

$\mathrm{Ca} 2+$ seems to be an essential metabolite for the dopamine hypothesis in SCZ, especially considering its crucial role in the function of dopamine receptors D1 and D2. ${ }^{38)}$ In most of the proteome analyses we performed in SCZ brain tissue, we observed the differential expression of $\mathrm{Ca} 2+$ homeostasis-related proteins. For instance, Calcineurin, that regulates dopaminergic ${ }^{39)}$ and glutamatergic ${ }^{40)}$ neurotransmission, which are frequently compromised in SCZ. ${ }^{41,42)}$ Dopaminergic hyperactivity in SCZ may result in altered N-methyl-D-aspartic acid (NMDA) receptor activation, which can lead to excitotoxicity and excess $\mathrm{Ca} 2+$ influxes through NMDA receptors. ${ }^{43)}$ Other important $\mathrm{Ca} 2+$-regulated proteins differentially expressed in SCZ are calmodulin and PMCA-4 which can interfere in the activity of phospholipase A2 (PLA2) leading to impairments on dopaminergic activity. In addition, myelinization dysfunction can also be triggered as a result of differential $\mathrm{Ca} 2+$ buffering. $^{44)}$

\section{Oligodendrocyte Metabolism}

The main role of oligodendrocytes in the central nervous system is to insulate axons with myelin, providing an electrically-insulating phospholipid layers that facilitate axonal signaling by increasing the speed at which the electrical impulses are propagated and by preventing the elec- 


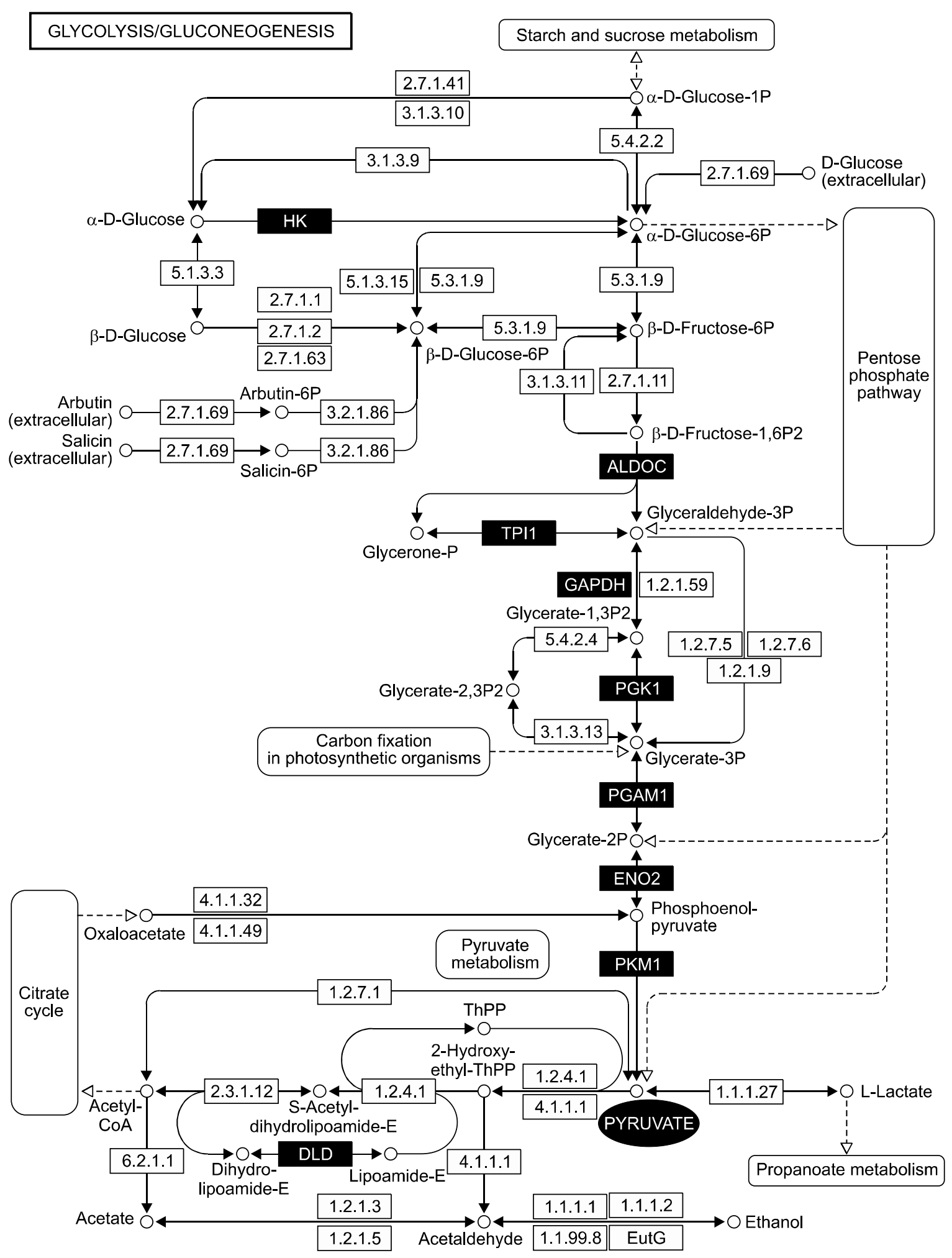

Fig. 1. Glucose metabolic pathway. The black boxes show the differentially expressed enzymes and metabolites revealed by proteomics in the glycolysis found in SCZ brain tissue (lllustration by KEGG - http://www.genome.jp/kegg). ${ }^{37}$ ) HK, hexokinase; ALDOC, fructosebisphosphate aldolase C; TPII, triosephosphate isomerase; GAPDH, glyceraldehyde-3-phosphate dehydrogenase; PGK1, phosphoglycerate kinase 1; PGAM1, phosphoglycerate mutase 1; ENO2, gamma-enolase; PKM1, pyruvate kinase isozymes M1/M2; DLD, dihydrolipoyl dehydrogenase, mitochondrial. 
trical current from leaving the axon. Additional functions of oligodendrocytes include growth factor synthesis, support to neuronal survival, as well as roles in neurodevelopment and neurotransmission. ${ }^{45,46)}$ Defects in myelin insulation may lead to ion leakage, which will reduce nerve impulse propagation. As a consequence, neuronal and glial functions will be compromised. Different research fields such as imaging, biochemical, epigenetic and neurodevelopment studies ${ }^{47-50)}$ have agreed that the dysfunction of oligodendrocytes is a pivotal feature of SCZ pathogenesis regarding brain connectivity. Several transcriptome studies using cDNA microarrays in SCZ brain tissue have supported oligodendrocyte dysfunction in $\mathrm{SCZ},{ }^{51)}$ so as proteomics. ${ }^{52)}$

2',3'-cyclic nucleotide 3' phosphodiesterase (CNP) was not only found to be differentially expressed in 6 transcriptome analyses but also in 3 proteome analyses of SCZ brain tissue. This protein binds to tubulins - which were also found to be differentially expressed in SCZ brains - to regulate the microtubule distribution in the cytoplasm. ${ }^{53)}$ Moreover, CNP promotes in oligodendrocytes microtubule assembly for process outgrowth and play indispensable roles in axonogenesis, RNA metabolic process, and synaptic transmission. ${ }^{54,55)}$ In addition, studies have confirmed genetic association of CNP with SCZ. ${ }^{56)}$ Transferrin, also found to be differentially expressed in a number of transcriptome and proteome analyses, has a central role in oligodendrocytes during myelination. ${ }^{57)}$

The major constituent of the myelin sheath of oligodendrocytes and Schwann cells is myelin basic protein (MBP). Transcriptome and proteome studies found MBP differentially expressed as well as myelin oligodendrocyte protein (MOG), supporting disturbances in myelinization. MBP and MOG are known markers for neurodegenerative diseases such as multiple sclerosis, suggesting that the altered levels of both proteins could be an indication of a kind of neurodegenerative event, as hypothesized previously. ${ }^{58)}$ Moreover, calcium imbalance, which was also observed by proteomics in SCZ, might lead to degenerative processes, as a result of dysfunctions in intracellular calcium buffering, storage and influx. ${ }^{59)}$

Regarding the biomarker point of view, we also found relatively different amounts of MOG and MBP in the CSF of SCZ patients, ${ }^{24)}$ suggesting them as potential biomarker candidates. $^{60)}$

\section{Cytoskeleton}

In our studies, we found a consistent dysregulation in the expression of cytoskeleton proteins (CPs). CPs pat- terns of expression are tissue specific and can directly influence in brain tissue processes such as cellular shaping, structural polarity, neuritogenesis, and neurotransmission.

Tubulin subunits were the most represented CPs in our studies. They are involved in several cellular activities such as mitosis, cytokinesis, and vesicular transport. ${ }^{61)}$ The classical astrocytic marker glial fibrillary acidic protein (GFAP) was consistently found to be differentially expressed in SCZ brains. GFAP is the major intermediate filament of mature astrocytes, which have pivotal role in the glutamatergic transmission. The dysfunction of astrocytes has been presented as a core feature of $\mathrm{SCZ},{ }^{62)}$ and alterations in GFAP expression may compromise synaptic functioning and behavior. ${ }^{63)}$ Dynamin 1 (DNM1) has also been reported consistently different in SCZ brains. This microtubule-binding protein plays a central role as a mechanochemical enzyme in synapses ${ }^{64)}$ as well as dopaminergic and glutamatergic transmission. ${ }^{65)}$ In addition, the neurofilaments $\mathrm{M}$ and $\mathrm{L}$ had their genes mapped to chromosomal regions related to SCZ susceptibility. These proteins play roles in the dopamine receptor signal transduction pathway. ${ }^{38)}$

\section{Final Remarks}

All SCZ proteome studies our group performed were done using brain tissue from patients treated with antipsychotic drugs. Therefore, we had already been asked whether some of the presented findings could be due drug treatment. Although we cannot rule out this possibility, this source of bias may be partially obviated by experimental evidence suggesting that the alterations of energy metabolism described in SCZ is a component of the disease itself and not an effect of antipsychotic drugs. ${ }^{66)}$ Moreover, the analyzed patients were under different therapeutic regimens. Thus, the identification of the same targets in such different samples supports the notion that the identified proteins are disease-associated. In addition, most of the identified proteins have not been reported to be associated with drug treatment, and some candidates also were found to be drug-effect independent by genetic linkage studies.

In sum, I feel safe to assume that the findings presented here reinforce the interest in the study of certain pathways and stimulate the study of the new potential markers identified by proteomics.

\section{Acknowledgments}

This article is a tribute to all schizophrenia patients and their families, who comprehend how important is their 
support for the elucidation of this disorder. Moreover, I thank all Professors and colleagues that have been sharing the path of studying schizophrenia with me, in special Prof. Emmanuel Dias-Neto, and Prof. Wagner Gattaz from University of São Paulo (USP, Brazil); Prof. Sergio Marangoni and Prof. Jose Novello from State University of Campinas (UNICAMP, Brazil); Prof. Chris Turck and Dr. Giuseppina Maccarrone from the Max Planck Institute of Psychiatry (Germany); Prof. Andrea Schmitt and Prof. Peter Falkai from University of Goettingen (Germany). I also thank Laura Pinheiro-Borges (BSc) for the unconditional support.

\section{REFERENCES}

1. Freedman R. Schizophrenia. N Engl J Med 2003;349:17381749.

2. Gejman PV, Sanders AR, Duan J. The role of genetics in the etiology of schizophrenia. Psychiatr Clin North Am 2010;33:35-66.

3. Barch DM. The cognitive neuroscience of schizophrenia. Annu Rev Clin Psychol 2005;1:321-353.

4. Miller EK, Cohen JD. An integrative theory of prefrontal cortex function. Annu Rev Neurosci 2001;24:167-202.

5. Steriade M, Llinás RR. The functional states of the thalamus and the associated neuronal interplay. Physiol Rev 1988;68: 649-742.

6. Andreasen NC, Arndt S, Swayze V 2nd, Cizadlo T, Flaum M, O'Leary D, et al. Thalamic abnormalities in schizophrenia visualized through magnetic resonance image averaging. Science 1994;266:294-298.

7. Luu P, Posner MI. Anterior cingulate cortex regulation of sympathetic activity. Brain 2003;126:2119-2120.

8. Benes FM. Neurobiological investigations in cingulate cortex of schizophrenic brain. Schizophr Bull 1993;19:537549.

9. Carter CS, MacDonald AW 3rd, Ross LL, Stenger VA. Anterior cingulate cortex activity and impaired self-monitoring of performance in patients with schizophrenia: an eventrelated fMRI study. Am J Psychiatry 2001;158:1423-1428.

10. Benes FM, Bird ED. An analysis of the arrangement of neurons in the cingulate cortex of schizophrenic patients. Arch Gen Psychiatry 1987;44:608-616.

11. Deakin JF, Simpson MD. A two-process theory of schizophrenia: evidence from studies in post-mortem brain. $J$ Psychiatr Res 1997;31:277-295.

12. Bogerts B. Recent advances in the neuropathology of schizophrenia. Schizophr Bull 1993;19:431-445.

13. Marini A, Spoletini I, Rubino IA, Ciuffa M, Bria P, Martino$\mathrm{tti} \mathrm{G}$, et al. The language of schizophrenia: an analysis of micro and macrolinguistic abilities and their neuropsychological correlates. Schizophr Res 2008;105:144-155.

14. Antonova E, Kumari V, Morris R, Halari R, Anilkumar A, Mehrotra $\mathrm{R}$, et al. The relationship of structural alterations to cognitive deficits in schizophrenia: a voxel-based morphometry study. Biol Psychiatry 2005;58:457-467.

15. Martí-Bonmatí L, Lull JJ, García-Martí G, Aguilar EJ, Moratal-Pérez D, Poyatos C, et al. Chronic auditory hallucinations in schizophrenic patients: MR analysis of the coincidence between functional and morphologic abnormalities. Radiology 2007;244:549-556.
16. Wilkins MR, Pasquali C, Appel RD, Ou K, Golaz O, Sanchez JC, et al. From proteins to proteomes: large scale protein identification by two-dimensional electrophoresis and amino acid analysis. Biotechnology (NY) 1996;14:61-65.

17. O'Farrell PH. High resolution two-dimensional electrophoresis of proteins. J Biol Chem 1975;250:4007-4021.

18. Martins-De-Souza D, Dias-Neto E, Schmitt A, Falkai P, Gormanns P, Maccarrone G, et al. Proteome analysis of schizophrenia brain tissue. World J Biol Psychiatry 2010;11: 110-120.

19. Gygi SP, Corthals GL, Zhang Y, Rochon Y, Aebersold R. Evaluation of two-dimensional gel electrophoresis-based proteome analysis technology. Proc Natl Acad Sci U S A 2000;97:9390-9395.

20. Link AJ, Eng J, Schieltz DM, Carmack E, Mize GJ, Morris $\mathrm{DR}$, et al. Direct analysis of protein complexes using mass spectrometry. Nat Biotechnol 1999;17:676-682.

21. Domon B, Aebersold R. Options and considerations when selecting a quantitative proteomics strategy. Nat Biotechnol 2010;28:710-721.

22. Maccarrone G, Turck CW, Martins-de-Souza D. Shotgun mass spectrometry workflow combining IEF and LC-MALDITOF/TOF. Protein J 2010;29:99-102.

23. Michalski A, Cox J, Mann M. More than 100,000 detectable peptide species elute in single shotgun proteomics runs but the majority is inaccessible to data-dependent LC-MS/MS. J Proteome Res 2011;10:1785-1793.

24. Martins-de-Souza D, Maccarrone G, Wobrock T, Zerr I, Gormanns P, Reckow S, et al. Proteome analysis of the thalamus and cerebrospinal fluid reveals glycolysis dysfunction and potential biomarkers candidates for schizophrenia. J Psychiatr Res 2010;44:1176-1189.

25. McLoughlin GA, Ma D, Tsang TM, Jones DN, Cilia J, Hill $\mathrm{MD}$, et al. Analyzing the effects of psychotropic drugs on metabolite profiles in rat brain using $1 \mathrm{H}$ NMR spectroscopy. J Proteome Res 2009;8:1943-1952.

26. Ditzen C, Varadarajulu J, Czibere L, Gonik M, Targosz BS, Hambsch B, et al. Proteomic-based genotyping in a mouse model of trait anxiety exposes disease-relevant pathways. Mol Psychiatry 2010;15:702-711.

27. Martins-de-Souza D, Schmitt A, Röder R, Lebar M, Schneider-Axmann T, Falkai P, et al. Sex-specific proteome differences in the anterior cingulate cortex of schizophrenia. J Psychiatr Res 2010;44:989-991.

28. Martins-de-Souza D, Gattaz WF, Schmitt A, Maccarrone G, Hunyadi-Gulyás E, Eberlin MN, et al. Proteomic analysis of dorsolateral prefrontal cortex indicates the involvement of cytoskeleton, oligodendrocyte, energy metabolism and new potential markers in schizophrenia. J Psychiatr Res 2009;43: 978-986.

29. Martins-de-Souza D, Gattaz WF, Schmitt A, Novello JC, Marangoni S, Turck $\mathrm{CW}$, et al. Proteome analysis of schizophrenia patients Wernicke's area reveals an energy metabolism dysregulation. BMC Psychiatry 2009;9:17.

30. Martins-de-Souza D, Gattaz WF, Schmitt A, Rewerts C, Maccarrone G, Dias-Neto E, et al. Prefrontal cortex shotgun proteome analysis reveals altered calcium homeostasis and immune system imbalance in schizophrenia. Eur Arch Psychiatry Clin Neurosci 2009;259:151-163.

31. Martins-de-Souza D, Gattaz WF, Schmitt A, Rewerts C, Marangoni S, Novello JC, et al. Alterations in oligodendrocyte proteins, calcium homeostasis and new potential markers in schizophrenia anterior temporal lobe are revealed by shotgun proteome analysis. J Neural Transm 2009;116:275-289. 
32. Kooy FH. Hyperglycemia in mental disorders. Brain 1919; 42:214-289.

33. Martins-de-Souza D, Harris LW, Guest PC, Bahn S. The role of energy metabolism dysfunction and oxidative stress in schizophrenia revealed by proteomics. Antioxid Redox Signal 2011;15:2067-2079.

34. Wolkin A, Jaeger J, Brodie JD, Wolf AP, Fowler J, Rotrosen $\mathrm{J}$, et al. Persistence of cerebral metabolic abnormalities in chronic schizophrenia as determined by positron emission tomography. Am J Psychiatry 1985;142:564-571.

35. Fucetola R, Newcomer JW, Craft S, Melson AK. Age- and dose-dependent glucose-induced increases in memory and attention in schizophrenia. Psychiatry Res 1999;88:1-13.

36. Guest PC, Wang L, Harris LW, Burling K, Levin Y, Ernst $\mathrm{A}$, et al. Increased levels of circulating insulin-related peptides in first-onset, antipsychotic naïve schizophrenia patients. Mol Psychiatry 2010;15:118-119.

37. Kanehisa M, Goto S. KEGG: kyoto encyclopedia of genes and genomes. Nucleic Acids Res 2000;28:27-30.

38. Bergson C, Levenson R, Goldman-Rakic PS, Lidow MS. Dopamine receptor-interacting proteins: the $\mathrm{Ca}(2+)$ connection in dopamine signaling. Trends Pharmacol Sci 2003; 24:486-492.

39. Greengard P. The neurobiology of slow synaptic transmission. Science 2001;294:1024-1030.

40. Zeng H, Chattarji S, Barbarosie M, Rondi-Reig L, Philpot BD, Miyakawa T, et al. Forebrain-specific calcineurin knockout selectively impairs bidirectional synaptic plasticity and working/episodic-like memory. Cell 2001;107:617-629.

41. Seeman P. Dopamine receptors and the dopamine hypothesis of schizophrenia. Synapse 1987;1:133-152.

42. Carlsson A, Waters N, Holm-Waters S, Tedroff J, Nilsson $\mathrm{M}$, Carlsson ML. Interactions between monoamines, glutamate, and GABA in schizophrenia: new evidence. Annu Rev Pharmacol Toxicol 2001;41:237-260.

43. Lee JM, Zipfel GJ, Choi DW. The changing landscape of ischaemic brain injury mechanisms. Nature 1999;399(6738 Suppl):A7-A14

44. Fu Y, Wang H, Huff TB, Shi R, Cheng JX. Coherent anti-Stokes Raman scattering imaging of myelin degradation reveals a calcium-dependent pathway in lyso-PtdCho-induced demyelination. J Neurosci Res 2007;85:2870-2881.

45. Du Y, Dreyfus CF. Oligodendrocytes as providers of growth factors. J Neurosci Res 2002;68:647-654.

46. Deng W, Poretz RD. Oligodendroglia in developmental neurotoxicity. Neurotoxicology 2003;24:161-178.

47. Kubicki M, Park H, Westin CF, Nestor PG, Mulkern RV, Maier SE, et al. DTI and MTR abnormalities in schizophrenia: analysis of white matter integrity. Neuroimage 2005;26:1109-1118.

48. Schmitt A, Wilczek K, Blennow K, Maras A, Jatzko A, Petroianu G, et al. Altered thalamic membrane phospholipids in schizophrenia: a postmortem study. Biol Psychiatry 2004; 56:41-45.

49. Davis KL, Stewart DG, Friedman JI, Buchsbaum M, Harvey $\mathrm{PD}$, Hof PR, et al. White matter changes in schizophrenia: evidence for myelin-related dysfunction. Arch Gen Psychiatry 2003;60:443-456.

50. Honer WG, Falkai P, Chen C, Arango V, Mann JJ, Dwork
AJ. Synaptic and plasticity-associated proteins in anterior frontal cortex in severe mental illness. Neuroscience 1999; 91:1247-1255.

51. Martins de Souza D, Dias-Neto E. RNA Biomarkers in Schizophrenia. In: Turck CW, editor. Biomarkers for Psychiatric Disorders, Vol. 1. Munich: Springer; 2009. p.97127.

52. Martins-de-Souza D. Proteome and transcriptome analysis suggests oligodendrocyte dysfunction in schizophrenia. $J$ Psychiatr Res 2010;44:149-156.

53. Bifulco M, Laezza C, Stingo S, Wolff J. 2',3'-Cyclic nucleotide 3'-phosphodiesterase: a membrane-bound, microtubuleassociated protein and membrane anchor for tubulin. Proc Natl Acad Sci U S A 2002;99:1807-1812.

54. Boccaccio GL, Colman DR. Myelin basic protein $m R N A$ localization and polypeptide targeting. J Neurosci Res 1995;42:277-286.

55. Sprinkle TJ, Lanclos KD, Lapp DF. Assignment of the human 2',3'-cyclic nucleotide 3'-phosphohydrolase gene to chromosome 17. Genomics 1992;13:877-880.

56. Peirce TR, Bray NJ, Williams NM, Norton N, Moskvina V, Preece A, et al. Convergent evidence for 2',3'-cyclic nucleotide 3'-phosphodiesterase as a possible susceptibility gene for schizophrenia. Arch Gen Psychiatry 2006;63:18-24.

57. Connor JR. Iron acquisition and expression of iron regulatory proteins in the developing brain: manipulation by ethanol exposure, iron deprivation and cellular dysfunction. Dev Neurosci 1994;16:233-247.

58. Lieberman JA. Is schizophrenia a neurodegenerative disorder? A clinical and neurobiological perspective. Biol Psychiatry 1999;46:729-739.

59. Wojda U, Salinska E, Kuznicki J. Calcium ions in neuronal degeneration. IUBMB Life 2008;60:575-590.

60. Martins-de-Souza D. Is the word 'biomarker' being properly used by proteomics research in neuroscience? Eur Arch Psychiatry Clin Neurosci 2010;260:561-562.

61. Benitez-King G, Ramírez-Rodríguez G, Ortíz L, Meza I. The neuronal cytoskeleton as a potential therapeutical target in neurodegenerative diseases and schizophrenia. Curr Drug Targets CNS Neurol Disord 2004;3:515-533.

62. Bernstein HG, Steiner J, Bogerts B. Glial cells in schizophrenia: pathophysiological significance and possible consequences for therapy. Expert Rev Neurother 2009;9:10591071.

63. Steffek AE, McCullumsmith RE, Haroutunian V, MeadorWoodruff JH. Cortical expression of glial fibrillary acidic protein and glutamine synthetase is decreased in schizophrenia. Schizophr Res 2008;103:71-82.

64. Sweitzer SM, Hinshaw JE. Dynamin undergoes a GTPdependent conformational change causing vesiculation. Cell 1998;93:1021-1029.

65. Dale LB, Babwah AV, Ferguson SS. Mechanisms of metabotropic glutamate receptor desensitization: role in the patterning of effector enzyme activation. Neurochem Int 2002;41:319-326.

66. Ben-Shachar D. The interplay between mitochondrial complex I, dopamine and Sp1 in schizophrenia. J Neural Transm 2009;116:1383-1396. 